BULL. AUSTRAL. MATH. SOC.

VOL. 12 (1975), 383-390.

\title{
Compatible tight Riesz orders on groups of integer-valued functions
}

\section{Gary Davis}

A construction due to Reilly is extended to show that there is a correspondence between compatible tight Riesz orders on $Z^{X}$ and non-principal filters on $X$. The maximal compatible tight Riesz orders are in one-to-one correspondence with non-principal ultrafilters, and are dual prime subsets of the positive set of $Z^{X}$. Conversely every dual prime algebraic Riesz order is maximal.

The lattice-ordered group $Z^{X}$ of all functions defined on the set $X$ and taking values in the totally-ordered group of rational integers $Z$ admits no compatible tight Riesz order when $X$ is finite. This can be seen by induction or, more conceptually the fact that $z^{X}$ then has no order-dense homomorphic image, and also from the fact that when $X$ is finite all ultrafilters on $X$ are principal. When $X$ is infinite, however, there are compatible tight Riesz orders on $Z^{X}:$ in the countably infinite case Reilly [4] has a construction that gives a compatible tight Riesz order for each non-principal ultrafilter on $X$.

For the definition of a compatible tight Riesz order on a latticeordered group see Wirth [5], where the following characterization occurs: a subset $T$ of an abelian lattice-ordered group $(G, \leqslant)$ is the strict positive set for a compatible tight Riesz order on $G$ if and only if the following three conditions are satisfied:

(1) $T$ is a proper dual ideal of $G^{+}=\{g \in G: g \geqslant 0\}$;

Received 24 January 1975. 
(2) $T=T+T$;

(3) if $0 \leqslant n x \leqslant y$ for all $y \in T$, for all integers $n \geq 1$, then $x=0$.

Throughout this paper a compatible tight Riesz order on $Z^{X}$ will be identified with its strict positive set.

The positive set of $z^{X}$ (namely, the set of $f \in Z^{X}$ satisfying $f(x) \geq 0$ for all $x \in X)$ is denoted by $Z_{+}^{X}$, and that of $Z$ by $Z_{+}$.

We define the mapping $\phi: Z^{X} \times Z \rightarrow z^{X}$ by $\phi(f, m)=(|f|-m) \vee 0$, where $|f|$ is the function $x+|f(x)|$ and $m$ also denotes the constant function whose value at each point of $X$ is $m \in Z$. For each $f \in Z^{X}$ the zero set of $f$ is $Z(f)=\{x \in X: f(x)=0\}$. The complement $X \backslash Z(f)$ of the zero set of $f$ is the support of $f$, denoted by $\operatorname{supp}(f)$.

LEMMA 1. If $(f, m),(g, n) \in Z_{+}^{X} \times Z_{+}$then

$$
\phi(f, m) \wedge \phi(g, n) \geq \phi(f \wedge g, \max (m, n))
$$

with equality if $m=n$.

Proof. For $(f, m),(g, n) \in Z_{+}^{X} \times Z_{+}$,

$$
\begin{aligned}
\phi(f, m) \wedge \phi(g, n)=((f-m) \vee 0) \wedge((g-n) \vee 0)=((f-m) \wedge(g-n)) \vee 0 \\
\geq((f-p) \wedge(g-p)) \vee 0=((f \wedge g)-p) \vee 0=\phi(f \wedge g, p)
\end{aligned}
$$

where $p=\max (m, n)$. If $m=n$ then

$$
\phi(f, n) \wedge \phi(g, n)=((f-n) \wedge(g-n)) \vee 0=((f \wedge g)-n) \vee 0=\phi(f \wedge g, n) .
$$

In the following result the term "adjunction" is used in the sense of Mac Lane [3] (and in preference to the equivalent "dual Galois correspondence").

THEOREM 2. There is an adjunction $\alpha \vdash \beta$ from the set of compatible tight Riesz orders on $\mathrm{Z}^{X}$ (ordered by inclusion) to the set of nonprincipal filters on $X$ (ordered by inclusion).

Proof. If $F$ is a non-principal filter on $X$ then $\beta(F)$ is defined to be the set $\left\{f \in Z_{+}^{X}: \operatorname{supp} \phi(f, m) \in F\right.$ for all $\left.m \in Z_{+}\right\}$. Since 
supp $(0,0)$ is empty the set $B(F)$ is properly contained in $Z_{+}^{X}$. Since $F$ is non-principal there is a sequence $S_{-1}, s_{0}, s_{1}, \ldots$ of subsets $S_{i} \in F$ with $S_{-1}=X, S_{i+1}$ properly contained in $S_{i}$, and $\bigcap_{i=0}^{\infty} S_{i}=\square$. If $f: X X \rightarrow Z$ is defined by $f(x)=n$ if $x \in S_{n-1} W_{n}$, $n \geq 0$, then $f$ is everywhere defined and

$$
\operatorname{supp} \phi(f, m)=\{x \in X: f(x)>m\}=\bigcup_{i=m-1}^{\infty} S_{i} \in F
$$

for all $m \in Z_{+}$, so that $\beta(F)$ is not empty.

If $f \in B(F)$ and $g \geq f$ then for each $m \in Z_{+}$, $\operatorname{supp} \phi(g, m) \supseteq \operatorname{supp} \phi(f, m) \in F$ so $\operatorname{supp} \phi(g, m) \in F$ and $g \in \beta(F)$. If $f, g \in \beta(F)$ and $m \in Z_{+}$then

$$
\operatorname{supp} \phi(f \wedge g, m)=\operatorname{supp} \phi(f, m) \wedge \phi(g, m)=\operatorname{supp} \phi(f, m) \cap \operatorname{supp} \phi(g, m) \in F
$$
so $f \wedge g \in B(F)$. That is, $B(F)$ is a proper dual ideal of $Z_{+}^{X}$.

The criterion (3) for $\beta(F)$ to be a compatible tight Riesz order is satisfied since $Z^{X}$ is archimedean. It remains to see that $B(F)=B(F)+B(F)$. One inclusion is immediate since

$$
\operatorname{supp} \phi(f+g, m) \geq \operatorname{supp} \phi(f, m) \cap \operatorname{supp} \phi(g, m)
$$

for all $f, g \in Z_{+}^{X}$ and all $m \in Z_{+}$. If, on the other hand, $f \in B(F)$ then we define $g \in Z^{X}$ by $g(x)=[f(x) / 2]+1$, where, for a rational number $\xi,[\xi]$ is the integral part of $\xi$. Suppose that $m \in Z_{+}$and $m \geq 2$. If $x \in \operatorname{supp} \phi(f, 2 m-2)$ then $f(x)>2 m-2$ so that $f(x) / 2>m-1$ and $[f(x) / 2] \geq m-1$. In this case $g(x) \geq m>m-1$ sc that $\operatorname{supp} \phi(f, 2 m-2) \subseteq \operatorname{supp} \phi(g, m-1)$ and, since $F$ is a filter, $\operatorname{supp} \phi(g, m-1) \in F$ for all $m \geq 2$. Then $\operatorname{supp}(g, 0) \supseteq \operatorname{supp}(g, 1) \in F$ so that $g \in \beta(F)$. Now we have to see that $h=f-g \in \beta(F)$. It follows, as above, that $\operatorname{supp} \phi(f, 2 m+2) \subseteq \operatorname{supp} \phi(h, m-1)$ for $m \geq 1$ so that $h \in B(F)$. This establishes $B(F)$ as a compatible tight Riesz order on 
$\mathrm{Z}^{X}$

Suppose that $T$ is a compatible tight Riesz order on $Z^{X}$ and $\alpha(T)=\left\{S \subseteq X: S \supseteq \operatorname{supp} \phi(f, m)\right.$ for some $\left.f \in T, m \in Z_{+}\right\}$. Then $\alpha(T)$ is a filter on $X$ since $\operatorname{supp} \phi(f, m) \cap \operatorname{supp} \phi(g, n)=\operatorname{supp} \phi(f, m) \wedge \phi(g, n) \supseteq \operatorname{supp} \phi(f \wedge g, \max (m, n))$ for $f, g \in Z_{+}^{X}$ and $m, n \in Z_{+}$. If $\alpha(T)$ is a principal filter then there is an $x \in X$ such that $f(x)>m$ for all $m \in Z_{+}$, which is absurd.

Finally we see that the mappings $\alpha, \beta$, which are clearly orderpreserving, provide us with an adjunction. Suppose that $\alpha(T) \subseteq F$, where $T$ is a compatible tight Riesz order and $F$ is a non-principal filter on $X$. If $f \in T$ then $\operatorname{supp} \phi(f, m) \in \alpha(T)$ for all $m \in Z_{+}$by definition of $\alpha(T)$, so $\operatorname{supp} \phi(f, m) \in F$ for all $m \in Z_{+}$. That is, $f \in B(F)$. On the other hand, suppose that $T \subseteq B(F)$. If $S \in \alpha(T)$ then $S \supseteq \operatorname{supp} \phi(f, m)$ for some $f \in T, m \in Z_{+}$. Since $f \in \beta(F)$ we have $\operatorname{supp} \phi(f, n) \in F$ for all $n \in Z_{+}$. In particular, $S \in F$ so we have $\alpha(T) \subseteq F$ if and only if $T \subseteq B(F)$.

We shall assume that the adjunction $\alpha+\beta$ between the set of compatible tight Riesz orders on $\mathrm{Z}^{X}$ and the set of non-principal filters on $X$ is the one described in Theorem 2 .

DEFINITION 3. A compatible tight Riesz order $T$ on $Z^{X}$ is prime if for all $f, g \in Z_{+}^{X}, f \vee g \in T$ implies $f \in T$ or $g \in T$. Further we say that $T$ is algebraic if $T=\beta \alpha(T)$ (of course, $T \subseteq \beta \alpha(T)$ in any case).

THEOREM 4. There is a one-to-one correspondence between nonprincipal ultrafilters on $X$ and maximal compatible tight Riesz orders on $\mathrm{Z}^{X}$. In particular, every maximal compatible tight Riesz order on $\mathrm{Z}^{X}$ is of the form $Z_{+}^{X} \backslash P_{U}$ where $P_{U}$ is a non-minimal prime subgroup of $Z^{X}$ defined in terms of the non-principal ultrafilter $u$, so every maximal compatible tight Riesz order is algebraic and prime. Conversely every 
prime algebraic compatible tight Riesz order is maximal.

Proof. The one-to-one correspondence between ultrafilters on $X$ and maximal compatible tight Riesz orders follows immediately from the existence of the adjunction $\alpha \vdash \beta$ so does the fact that maximal compatible tight Riesz orders are algebraic. If $U$ is a non-principal ultrafilter on $X$ then $P=\left\{f \in Z^{X}: Z \phi(f, m) \in U\right.$ for some $\left.m \in Z_{+}\right\}$is a proper convex sublattice subgroup of $z^{X}$ which is prime but not minimal prime since $P_{U}$ properly contains the prime subgroup $P_{0}=\left\{f \in Z^{X}: z(f) \in U\right\}$. Then $\beta(U)=\left\{f \in Z_{+}^{X}: \operatorname{supp} \phi(f, m) \in U\right.$ for all $\left.m \in Z_{+}\right\}=Z_{+}^{X} \backslash P_{U}$ and $B(U)$ is prime since $P_{U}$ is a join sublattice of $Z^{X}$.

Suppose conversely that $T$ is a prime algebraic compatible tight Riesz order on $Z^{X}$. We shall see that $\alpha(T)$ is a prime, and therefore maximal, filter on $X$. It is sufficient to show that if $A \cup B \in \alpha(T)$, where $A, B \subseteq X$ and $A \cap B=\square$, then $A \in \alpha(T)$ or $B \in \alpha(T)$. Suppose $A \cup B \supseteq \operatorname{supp} \phi(f, m)$ for some $f \in T, m \in Z_{+}$, but $A, B \mid \alpha(T)$. Then the sets $A^{\prime}=\{x \in A: f(x)>m\}, B^{\prime}=\{x \in B: f(x)>m\}$ are nonempty. We define $g, h: X \rightarrow Z$ as follows:

$$
g(x)=\left\{\begin{array}{lll}
0 & \text { if } & x \in A^{\prime} \\
f(x) & \text { if } & x \notin A^{\prime}
\end{array}, \quad h(x)=\left\{\begin{array}{ll}
0 & \text { if } x \in B^{\prime} \\
f(x) & \text { if } x \notin B^{\prime}
\end{array} .\right.\right.
$$

Then

$$
\operatorname{supp} \phi(g, m)=\{x \in X: g(x)>m\}=B^{\prime} \subseteq B
$$

and

$$
\operatorname{supp} \phi(h, m)=\{x \in X: h(x)>m\}=A^{\prime} \subseteq A
$$

Further, $g \vee h=f \in T$ so either $g \in T$ or $h \in T$ (since $g, h \in Z_{+}^{X}$ ). Thus $B \in \alpha(T)$ or $A \in \alpha(T)$. Since $\alpha(T)$ is a prime filter on $X$ it is an ultrafilter, and $T=\beta \alpha(T)$ is a maximal compatible tight Riesz order. 
COROLLARY 5. If $X$ has cardinality $k \geq N_{0}$ then there are $2^{2 k}$ maximal compatible tight Riesz orders on $Z^{X}$.

Proof. There are $2^{2^{k}}$ non-principal ultrafilters on $z^{X}$ (Bell and Slomson [1], p. 108).

COROLLARY 6. If $T$ is an algebraic tight Riesz order on $Z^{X}$ then $T=2_{+}^{X} \backslash U\left\{P_{U_{i}}: i \in I\right\}$ for some class $\left\{U_{i}: i \in I\right\}$ of non-principal ultrafizters on $X$.

For a given ultrafilter $U$ on $X$ the totally-ordered group $Z^{X} / P_{0}$ has the same first-order properties as $Z$, since this group is an ultrapower of $Z$. If $U$ is a non-principal ultrafilter the totally-ordered group $Z^{X} / P_{u}$ must be dense however, since $Z_{+}^{X} \backslash P_{u}$ is a compatible tight Riesz order.

THEOREM 7. Let $G$ be an abelian lattice-ordered group and $I$ a convex sublattice subgroup of $G$. Then $T=G^{+} \backslash I$ satisfies $T=T+T$ if and only if $G / I$ is a dense lattice-ordered group.

Proof. Suppose that $G / I$ is dense. If $t \in T$ and $t+I>0$ in $G / I$ then there is an $x \in G$ satisfying $t+I>x+I>0$. With $y=(x \vee 0) \wedge t$ we have $t>y>0$ so $y \in G^{+} \backslash I, t-y \in G^{+} \backslash I$ and $t=y+(t-y) \in T+T$. The reverse inclusion is immediate. On the other hand suppose that $T=T+T$ and $x+I>0$ in $G / I$. Then $x \in I$ and $x+y>0$ for some $y \in I$ so $x+y=t_{1}+t_{2}$ where $t_{1}, t_{2} \in T$. Then $0<t_{1}+I<x+I$ so $G / I$ is dense.

The problem of characterizing the groups $z^{X} / P_{u}$, for $u$ a nonprincipal ultrafilter on $X$ does not seem to be easy. Without something like Gödel's axiom of constructability even the cardinality of $Z^{X} / P_{U}$ is unclear. As a group we can write $\mathrm{Z}^{X} / P_{u}$ as $\left(\mathrm{Z}^{X} / P_{0}\right) / P_{U^{\prime}} / P_{0}$ and then use known results on the cardinality of ultrapowers in an attempt to determine 
the cardinality of $\mathrm{Z}^{X} / P_{U}$.

When $X$ is countable (non-finite) the cardinality of the ultrapower $2^{X} / P_{0}$ is $2^{\aleph_{0}}$, for each ultrafilter $u$ on $X$ (Bell and Slomson [1], p.129). As Reilly [4] has remarked the prime subgroup $P_{U}$ covers $P_{0}$ in $\mathrm{Z}^{X}$ so $P_{\mathrm{U}} / P_{0}$ is isomorphic with a subgroup of the real numbers.

The totallywrdered groups $\mathrm{Z}^{X} / P_{U}$ admit more interpolation than that implied by density. A result of Gillman and Jerison [2] (Lemma 13.7), is valid with the real numbers $\mathrm{R}$ replaced by $\mathrm{Z}$ and then says that if $A, B$ are countable subsets of $Z / P_{U}$ with $A<B$ then $A \leq g \leq B$ for some $g \in \mathrm{Z}^{X} / P$. We summarize these properties of $\mathrm{z}^{X} / P_{u}$ in the following statement.

PROPOSITION 8. If $X$ is a countable set and $U$ is a non-principal ultrafizter on $X$ then the group $G_{U}=Z^{X} / P_{U}$ has the following properties:

(1) $\aleph_{0} \leq\left|G_{u}\right| \leq 2^{\aleph_{0}}$;

(2) $G_{U}$ is dense;

(3) if $A, B \subseteq G,|A \cup B| \leq \kappa_{0}$ and $A<B$ then $A \leq g \leq B$ for some $g \in G_{U}$;

(4) $G_{U}$ is a quotient of an ultrapower of $Z$ by a real group.

\section{References}

[1] J.L. Bell and A.B. Slomson, Models and ultraproducts: an introduction (North-Holland, Amsterdam, London, 1969).

[2] Leonard Gillman and Meyer Jerison, Rings of continuous functions (Van Nostrand, Princeton, New Jersey; Toronto; London; New York; 1960). 
[3] S. Mac Lane, Categories for the working mathematician (Graduate Texts in Mathematics, 5. Springer-Verlag, New York, Heidelberg, Berlin, 1971).

[4] N.R. Reilly, "Compatible tight Riesz orders and prime subgroups", Glasgow Math. J. 14 (1973), 245-160.

[5] Andrew Wirth, "Compatible tight Riesz orders", J. Austral. Math. Soc. 15 (1973), 105-111.

Department of Mathematics,

La Trobe University,

Bundoora,

Victoria. 